\title{
Dry needling in stroke
}

\author{
Nirmal Surya ${ }^{1,2}{ }^{*}$, Guhan Ramamurthy ${ }^{3}$ (1) \\ ${ }^{1}$ Chairman Surya Neuro Centre Mumbai, President Indian Federation of Neurorehabilitation (IFNR), Mumbai 400020, India \\ ${ }^{2}$ Bombay Hospital and Medical Research Centre, Mumbai 400020, India \\ ${ }^{3}$ Department of Neurology and Neurorehabilitation, BG Hospital, Tiruchendur, Tuticorin 628216, India
}

*Correspondence: Nirmal Surya, Chairman Surya Neuro Centre Mumbai, President Indian Federation of Neurorehabilitation (IFNR), Mumbai 400020, India; Bombay Hospital and Medical Research Centre, Mumbai 400020, India.nirmal_surya@yahoo.com Academic Editor: Noureddin Nakhostin Ansari, Tehran University of Medical Sciences, Iran

Received: November 9, 2021 Accepted: December 23, 2021 Published: February 17, 2022

Cite this article: Surya N, Ramamurthy G. Dry needling in stroke. Explor Neuroprot Ther. 2022;2:28-35. https://doi. org/10.37349/ent.2022.00016

\begin{abstract}
Stroke causes acute neurological deficit which is an important cause of morbidity and mortality. Neurorehabilitation is an important dimension in the management of post-stroke deficits. Spasticity, pain, and neurological deficits are contributors to post-stroke disability. Dry needling (DN) is a technique commonly used in the management of myofascial pain. Recent evidence suggests its efficacy in the management of post-stroke disability. The descriptive review on the use of DN summarises the evidence for the management of post-stroke patients such as spasticity, balance, pain, functional outcome, tremor, and ultrasonographic evidence. The filiform needle is inserted into the target muscle until a local twitch response is obtained. The effects of DN are produced by the local stretch of the spastic muscle and afferent modulation of the reflex arc that decreases the excitability of the alpha motor neuron. The DN reduces muscle spasticity in post-stroke patients. The improved spasticity is translated to better functional outcomes and balance. The procedure is also shown to reduce pain including post-stroke shoulder pain. It is also shown to improve tremors in post-stroke patients. Ultrasonographic evidence of the beneficial effects of DN shows improved measures in the pennate angle and mean muscle thickness. Concurrent use of DN and electrical stimulation improve spasticity, the effect which may be seen for longer periods. DN is emerging as a useful and cost-effective technique in the management of post-stroke patients. The evidence for the use of DN in the management of post-stroke spasticity is high. However, more research is required to assess its efficacy in functional outcomes and other aspects of the stroke.
\end{abstract}

\section{Keywords}

Dry needling, spasticity, post-stroke pain, balance

\section{Introduction}

Stroke is a leading cause of disability worldwide. The disability resulting from stroke has health, social and economic implications for the patient. The recent advances in the acute management of stroke with the use of recanalization procedures have improved the recovery of patients with stroke. However, neurorehabilitation

(C) The Author(s) 2022. This is an Open Access article licensed under a Creative Commons Attribution 4.0 International License (https://creativecommons.org/licenses/by/4.0/), which permits unrestricted use, sharing, adaptation, distribution and reproduction in any medium or format, for any purpose, even commercially, as long as you give appropriate credit to the original author(s) and the source, provide a link to the Creative Commons license, and indicate if changes were made. 
remains an important dimension in the management and optimization of the functional status of patients with stroke who miss the window of opportunity to recanalization and in those who are left with residual deficits. The important contributors of disability in post-stroke patients include spasticity, post-stroke pain, and neurological deficits. The evidence for rehabilitation in stroke is ever-expanding [1].

The needle therapies in rehabilitation include wet and dry needling (DN). In wet needling, the bore in the needle is used to deliver drugs locally to the site, such as botulinum toxin injection, anesthetic drugs, steroids, sclerosing agents, and others, to produce the desired effect. In DN, a filiform needle without a bore is used akin to acupuncture without the delivery of any drugs [2]. However, it differs from acupuncture in indications, technique, and expertise level. DN is commonly used in the treatment of pain arising from musculoskeletal structures such as myofascial pain syndromes. The evidence for the use of DN in the management of spasticity due to various neurological conditions such as stroke has been supportive. Recently evidence for the use of DN in the management of stroke other than spasticity has also been emerging. In this descriptive review, we summarise the various evidence that supports or refutes the use of DN in stroke. DN is a skilled interventional procedure that can easily be performed by physical therapists, chiropractors, acupuncturists who are trained to perform DN [3].

\section{Elements and techniques of DN}

DN uses a technique that traverses the skin, subcutaneous tissue, fascia, and muscle depending on the technique of DN. The elements of DN include [4, 5]:

1. Use of the aseptic technique.

2. The diameter of the filiform needle used ranges between $0.012 \mathrm{~mm}$ and $0.35 \mathrm{~mm}$.

3. The needle is of adequate length to reach the target site depending on the technique used.

The DN may be categorized into superficial and deep DN. In superficial DN, the needle is inserted 5-10 $\mathrm{mm}$ depth and comes to lie above the trigger points. The duration of the retention may vary between $30 \mathrm{~s}$ to $3 \mathrm{~min}$ and the procedure may be repeated if the pain persists. The needle may be superficially inserted, perpendicularly or at an angle of 20-30 degrees and different manipulations may be performed. In deep DN, the needles are inserted to reach the trigger points using the 'pistoning technique' (fast in and out technique), or the needle may be retained for varying periods of time. The needle in deep DN is inserted perpendicularly or at an angle of 30-45 degrees. The needle must be inserted, twisted, and pulled until a local twitch response is obtained that suggests proper technique. The procedure can be performed under ultrasound guidance especially in the muscle groups that are not superficially palpable $[2,5,6]$.

\section{Mechanism of DN}

The proposed mechanism through which DN produces benefits is speculative. The effects of DN are produced by inducing a stretch while within the spastic tissue, increasing the distance between Z-lines in the sarcomere thereby reducing the overlap between the contractile proteins and reducing the tone. The DN may also decrease the firing of the afferent pathway originating in the muscle to the spinal cord and thereby modulates the activity of the motor neuron in the stretch reflex and decreases its tone. This is evident from the local twitch response elicited by the DN. Overall it decreases the activity of the stretch reflex and the alpha motor neuron $[7,8]$.

DN causes the inactivation of trigger points in the target muscle. It reduces the central pain sensitization, allodynia associated with local pain, and hyperalgesia associated with referred pain. In the stimulated muscle, there is augmented blood flow and oxygenation that lasts beyond the period of DN. It also produces a chemical milieu with reduced Substance-P and calcitonin gene-related peptides that favors reducing pain. The DN increases the range of movement and improves muscle activation patterns [9, 10] (Figure 1). 


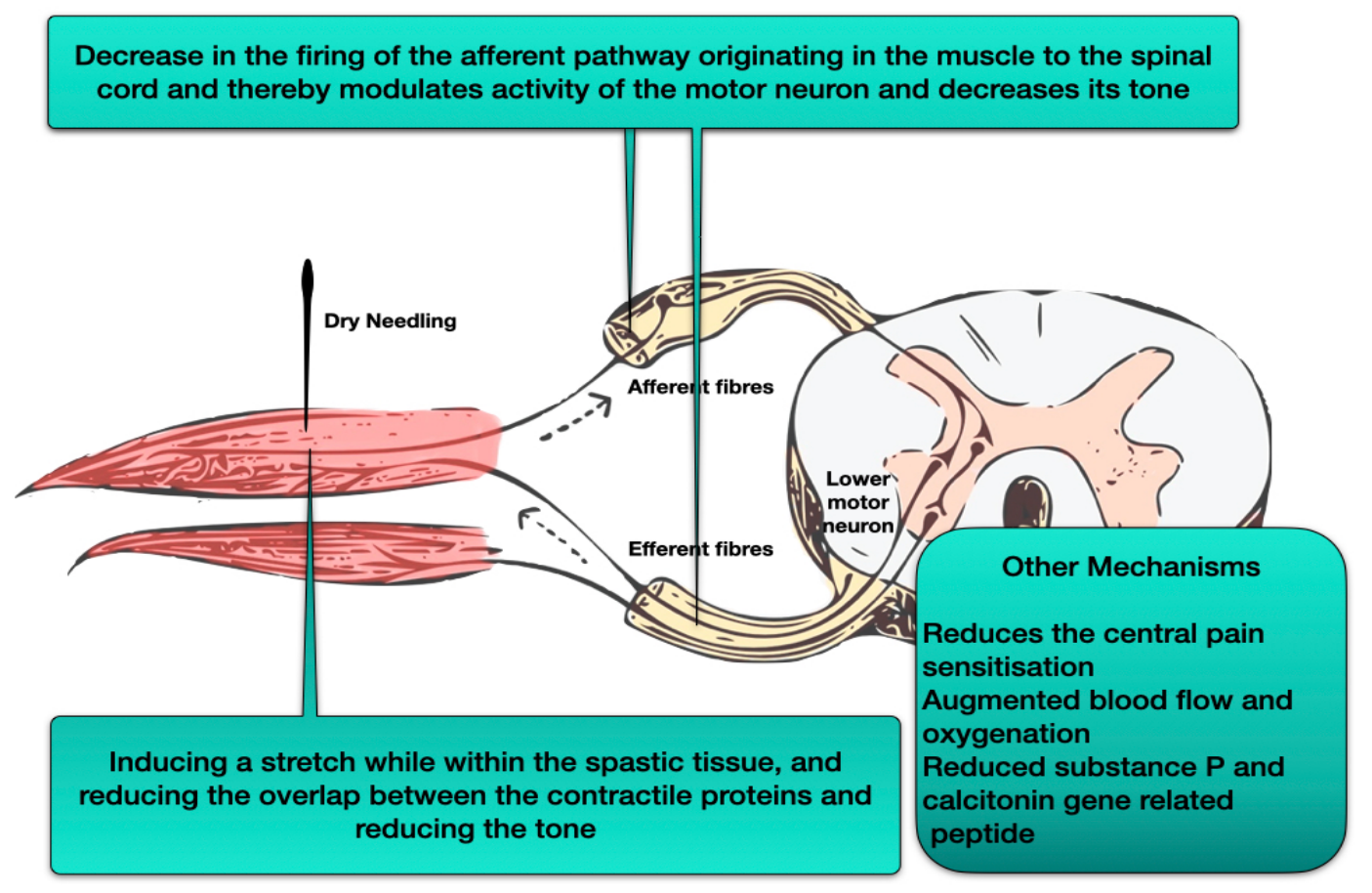

Figure 1. Mechanism of dry needling in post-stroke spasticity and pain

\section{DN in neurology}

DN is commonly used in the management of pain arising from musculoskeletal structures such as myofascial pain. The evidence for its use in spasticity and neuropathic pain arising from various neurological disorders has been promising. The initial reports of the use of deep DN in a patient with hypertonia due to spastic tetraparesis reduced the resistance to passive range of motion and improved the viscoelastic properties of the muscle [11]. Further, the effect of DN in hypertonia due to stroke in the subacute and chronic phases has been studied and now has been extended to study in patients with multiple sclerosis. In Parkinson's disease, the evidence for DN in improving the tone, gait, and activities of daily living are limited and further research is required in the area. In the following review, the role of DN in the multifaceted management of stroke patients has been summarised $[12,13]$.

\section{DN in stroke}

The rehabilitation of deficit in stroke patients is multimodal. A comprehensive approach is required to improve functional outcomes in these patients. DN is emerging as an important tool in the management of post-stroke deficits. The occurrence of increased myofascial trigger points in post-stroke patients may suggest that DN may serve as a useful option in the management of post-stroke patients [14]. DN is shown to improve spasticity, functional outcome, and balance in post-stroke patients. The technique and dosing employed in performing DN were heterogeneous across the studies. Most of the studies performed DN in chronic stroke patients.

\section{DN in post-stroke spasticity}

Spasticity is one of the major causes of disability in patients with stroke. Several pharmacological measures using muscle relaxants and interventional procedures such as intrathecal baclofen and botulinum toxin injection are used in the management of spasticity. DN is emerging as a useful technique in the management of post-stroke spasticity.

The study by Ghannadi et al. [15] assessed the effects of DN in the improvement of lower limb functions following stroke. The DN was performed using stainless steel needle in the gastrocnemius muscle using the fast in and out technique until the local twitch response is obtained and performed for 1 min for 3 sessions a 
week. The spasticity measured by the Modified Modified Ashworth Scale (MMAS) revealed a reduction in the spasticity in the interventional cohort.

In randomized control trial by Sánchez-Mila et al. [16], a similar technique as above was used, but the muscle target was tibialis posterior and was performed under ultrasound guidance in a single session. The interventional group consisted of DN in addition to the therapeutic sessions according to Bobath concept and the control group consisted of therapeutic sessions according to Bobath concept alone. The spasticity measured by MMAS showed a greater reduction in tone in the interventional group.

The systematic review on the effect of DN by Carusotto et al. [17] has shown that DN decreased spasticity when performed in the shoulder, arm, and flexors of the wrist and improved the range of motion and when performed in the gastrocnemius decreased tone and improved the gait. The meta-analysis and systematic review by Fernández-de-Las-Peñas et al. [18] showed a significant decrease in spasticity and tone in the interventional group. The decrease in the tone was more evident in the lower limb compared to the upper limb in the short-term follow-up. The quality of evidence is moderate.

In a study performed by Fakhari et al. [19], deep DN performed in wrist flexors for 2 min improved the spasticity immediately following the procedure and lasted for $1 \mathrm{~h}$ following the procedure. A similar result was observed in a case report published by Ansari et al. [20].

The study performed by Cuenca Zaldívar et al. [21] assessed the efficacy of DN in post-stroke patients when performed in the sub-acute phase. The interventional group received the deep DN on weeks 1, 2, 3, 4,6 , and 8 with an interval of 7 days between sessions. The technique of DN for hypertonia and spasticity was used with repeated insertion at a frequency of $1 \mathrm{~Hz}$ according to the tolerability of the patient with an intention to elicit a local twitch response. The patients in the interventional limb who received deep DN in addition to standard physiotherapy had a greater reduction in spasticity when compared to standard physiotherapy alone.

\section{DN in functional outcome and pain}

In the study by Ghannadi et al. [15], DN had improved the functional outcome measures of the lower limb following stroke as evidenced by improved metrics in the Timed Up and Go test, Ten-Meter Walk Test, and Barthel Index. There was also an increase in the range of motion demonstrated passively in the ankle joint. In randomized control trial by Zacarías Sánchez-Mila et al. [16], the functional outcome measured by sensory subscale and range of motion subscale of Fugl-Meyer Scale showed significant improvement in the interventional group which included the DN. However, these changes did not amount to the 'minimal clinically important difference' defined in the study. In the study by Hadi et al. [22], DN of the selected muscles improved the timing in the Timed Up and Go test that had translated to improved gait function. This shows that improvement in the spasticity is translated to improved functional outcomes in the patients.

The meta-analysis and systematic review by Fernández-de-Las-Peñas et al. [18] showed significantly decreased pain in the interventional group. The effect of DN in post-stroke shoulder pain was also evident but the quality of evidence was low. The DN also significantly increased the pressure pain threshold in the interventional limb. The use of DN in post-stroke shoulder pain was based on the existence of trigger points in the musculature surrounding the shoulder joint. The trigger points were elicited in the supraspinatus, infraspinatus, upper trapezius, paraspinal musculature, and splenius capitis. The effect of the reduction in pain is mediated through central and peripheral mechanisms.

\section{DN in balance}

In the study by Ghannadi et al. [15], DN had improved the balance of post-stroke patients. This was evident from the longest single leg stance. In the randomized control trial by Sánchez-Mila et al. [16], the functional outcome measured by the balance subscale of the Fugl-Meyer Scale showed significant improvement in the interventional group which included the DN. A similar finding was reported in the measurement using dynamic posturography. Dynamic posturography provides a sensitive measure of balance in comparison to 
clinical scales and measures. The measures of balance and accuracy in dynamic posturography improved following DN of the unilaterally involved tibialis posterior muscle [16].

\section{DN in tremor}

In a case report published by Ortín et al. [23], the use of DN for the treatment of post-stroke tremor involving the myofascial trigger points of the muscles supraspinatus, infraspinatus, upper trapezius, mid-region of the deltoid, teres minor, biceps brachii short head, triceps brachii long head, brachioradialis, extensor digitorum, flexor digitorum profundus, and flexor digitorum superficialis was beneficial as evident in the clinical, electrophysiological and functional outcomes of the tremor. The patient had improved postural and functional components of tremor [23].

\section{Imaging evidence in DN}

Ultrasonographic evaluation of the affected muscle is a cost-effective method to assess the architecture of the muscle. The angle between the pennation of the fibers, the mean muscle thickness, and the muscle length are sonographic measures to assess the capability of the muscle to generate force. A decrease in the pennation angle, mean muscle thickness, and increased muscle length suggests improved muscle function. In the study by Ghannadi et al. and Hadi et al. [15, 22], DN in the gastrocnemius muscle has shown a favorable reduction in the pennation angle and the mean muscle thickness and increase in the muscle length that correlates with an improved gait function (Table 1).

Table 1. The efficacy of DN in spasticity, post-stroke pain, functional outcome, balance, tremor, and imaging measures

\begin{tabular}{ll}
\hline Parameter & Efficacy \\
\hline Spasticity & Improved spasticity as measured by MMAS \\
$\begin{array}{l}\text { Post-stroke pain } \\
\text { Functional outcome }\end{array}$ & $\begin{array}{l}\text { Reduced post-stroke pain including post-stroke shoulder pain (low evidence) } \\
\text { Improvement in Timed Up and Go test, Ten Meter Walk Test, Barthel Index, and passive range } \\
\text { Improved outcome in sensory and range of motion subscales in Fugl-Meyer Scale }\end{array}$ \\
Balance & $\begin{array}{l}\text { Improved single-leg stance } \\
\text { Improved balance subscale in Fugl-Meyer Scale }\end{array}$ \\
& $\begin{array}{l}\text { Improved balance and accuracy in dynamic posturography } \\
\text { Improved postural and functional components of tremor }\end{array}$ \\
Muscular architecture & Decrease in the pennation angle and mean muscle thickness \\
\hline
\end{tabular}

\section{DN with concurrent electrical stimulation}

DN can be performed as a combination in multimodal rehabilitation approaches such as DN with concurrent electrical stimulation. However, the evidence is limited. Electrical stimulation causes muscle contraction and provides visual and sensory feedback to the patient. The electrical stimulation is delivered using surface electrodes. In a meta-analysis, it is shown to improve motor function. Ghaffari et al. [24] have shown that the combination is shown to decrease spasticity, increase the power as assessed by the Medical Research Council (MRC) manual muscle testing scale in the wrist extensors and improve functional outcome as measured by Brunstrom hand functional recovery after a single session. These effects were shown to be persistent at 1 month.

\section{Adverse events}

The most common side effect noted following DN is post-interventional soreness. The other minor effects noted following DN include pain, bruising, and bleeding. The major side effects are rare and include loss of consciousness. Hence DN appears to be a safe intervention while performed by trained personnel [18]. 


\section{Conclusions}

The evidence in the early years of DN in the management of patients with stroke appears as a promising tool in the comprehensive therapeutic armamentarium in the rehabilitation of stroke patients. The evidence for the use of DN in the management of post-stroke spasticity is high. It is also found to improve functional outcome, balance, gait, and decrease post-stroke pain. However, more research is required to assess its efficacy in functional outcomes and other aspects of the stroke. DN is a cost-effective and easy-to-use method in the management of post-stroke patients [25].

\section{Abbreviations}

DN: dry needling

MMAS: Modified Modified Ashworth Scale

\section{Declarations}

\section{Author contributions}

NS: conception of the article, design of the work, literature review and search, organization, analysis and interpretation of the literature and data, manuscript write up and manuscript revision. GR: literature review and search, organization, analysis and interpretation of the literature and data, manuscript write up, and manuscript revision. Both authors contributed to manuscript revision, read and approved the submitted version.

\section{Conflicts of interest}

The authors declare that they have no conflicts of interest.

\section{Ethical approval}

Not applicable.

\section{Consent to participate}

Not applicable.

\section{Consent to publication}

Not applicable.

\section{Availability of data and materials}

Not applicable.

\section{Funding}

Not applicable.

\section{Copyright}

(C) The Author(s) 2022.

\section{References}

1. Brewer L, Horgan F, Hickey A, Williams D. Stroke rehabilitation: recent advances and future therapies. QJM. 2013;106:11-25.

2. Dunning J, Butts R, Mourad F, Young I, Flannagan S, Perreault T. Dry needling: a literature review with implications for clinical practice guidelines. Phys Ther Rev. 2014;19:252-65.

3. Pourahmadi M, Mohseni-Bandpei MA, Keshtkar A, Koes BW, Fernández-de-Las-Peñas C, Dommerholt J, et al. Effectiveness of dry needling for improving pain and disability in adults with tension-type, cervicogenic, or migraine headaches: protocol for a systematic review. Chiropr Man Therap. 2019;27:43. 
4. Simons DG, Travell JG, Simons LS. Travell \& Simons' myofascial pain and dysfunction: upper half of body. Lippincott williams \& wilkins; 1999.

5. Zhou K, Ma Y, Brogan MS. Dry needling versus acupuncture: the ongoing debate. Acupunct Med. 2015;33:485-90.

6. Physical therapists \& the performance of dry needling [Internet]. APTA; c2012 [cited 2021 Nov 4]. Available from: https://www.mptalliance.com/wp-content/uploads/2015/12/APTA-PTs-and-DryNeedling-Resource-Paper.pdf

7. Cagnie B, Dewitte V, Barbe T, Timmermans F, Delrue N, Meeus M. Physiologic effects of dry needling. Curr Pain Headache Rep. 2013;17:348.

8. Chou LW, Kao MJ, Lin JG. Probable mechanisms of needling therapies for myofascial pain control. Evid Based Complement Alternat Med. 2012;2012:705327.

9. Cagnie B, Barbe T, De Ridder E, Van Oosterwijck J, Cools A, Danneels L. The influence of dry needling of the trapezius muscle on muscle blood flow and oxygenation. J Manipulative Physiol Ther. 2012;35:685-91.

10. Dommerholt J. Dry needling-peripheral and central considerations. J Man Manip Ther. 2011;19:223-7.

11. Gallego PH, del Moral OM. A case study looking at the effectiveness of deep dry needling for the management of hypertonia. J Musculoskelet Pain. 2007;15:55-60.

12. Motamedzadeh O, Nakhostin Ansari N, Naghdi S, Azimi A, Mahmoudzadeh A, Calvo S, et al. A study on the effects of dry needling in multiple sclerosis patients with spasticity: protocol of a randomized waitlist-controlled trial. JAMS. 2021;14:82-8.

13. Brandín-de la Cruz N, Calvo S, Rodríguez-Blanco C, Herrero P, Bravo-Esteban E. Effects of dry needling on gait and muscle tone in Parkinson's disease: a randomized clinical trial. Acupunct Med. 2022;40:3-12.

14. Villafañe JH, Lopez-Royo MP, Herrero P, Valdes K, Cantero-Téllez R, Pedersini P, et al. Prevalence of myofascial trigger points in poststroke patients with painful shoulders: a cross-sectional study. PM R. 2019;11:1077-82.

15. Ghannadi S, Shariat A, Ansari NN, Tavakol Z, Honarpishe R, Dommerholt J, et al. The effect of dry needling on lower limb dysfunction in poststroke survivors. J Stroke Cerebrovasc Dis. 2020;29:104814.

16. Sánchez-Mila Z, Salom-Moreno J, Fernández-de-Las-Peñas C. Effects of dry needling on post-stroke spasticity, motor function and stability limits: a randomised clinical trial. Acupunct Med. 2018;36:358-66.

17. Carusotto AF, Hakim RM, Oliveira RG, Piranio A, Coughlan CP, MacDonald TJ. Effects of dry needling on muscle spasticity in adults with neurological disorders: a systematic review. Phys Ther Rev. 2021;26:380-5.

18. Fernández-de-Las-Peñas C, Pérez-Bellmunt A, Llurda-Almuzara L, Plaza-Manzano G, De-la-Llave-Rincón AI, Navarro-Santana MJ. Is dry needling effective for the management of spasticity, pain, and motor function in post-stroke patients? A systematic review and meta-analysis. Pain Med. 2021;22:131-41.

19. Fakhari Z, Ansari NN, Naghdi S, Mansouri K, Radinmehr H. A single group, pretest-posttest clinical trial for the effects of dry needling on wrist flexors spasticity after stroke. NeuroRehabilitation. 2017;40:325-36.

20. Ansari NN, Naghdi S, Fakhari Z, Radinmehr H, Hasson S. Dry needling for the treatment of poststroke muscle spasticity: a prospective case report. NeuroRehabilitation. 2015;36:61-5.

21. Cuenca Zaldívar JN, Calvo S, Bravo-Esteban E, Oliva Ruiz P, Santi-Cano MJ, Herrero P. Effectiveness of dry needling for upper extremity spasticity, quality of life and function in subacute phase stroke patients. Acupunct Med. 2021;39:299-308.

22. Hadi S, Khadijeh O, Hadian M, Niloofar AY, Olyaei G, Hossein B, et al. The effect of dry needling on spasticity, gait and muscle architecture in patients with chronic stroke: a case series study. Top Stroke Rehabil. 2018;25:326-32.

23. Ortín JA, Bravo-Esteban E, Ibáñez J, Herrero P, Gómez-Soriano J, Marcén-Román Y. Effects of deep dry needling on tremor severity and functionality in stroke: a case report. Healthcare (Basel). 2021;9:5. 
24. Ghaffari MS, Shariat A, Honarpishe R, Hakakzadeh A, Cleland JA, Haghighi S, et al. Concurrent effects of dry needling and electrical stimulation in the management of upper extremity hemiparesis. J Acupunct Meridian Stud. 2019;12:90-4.

25. Fernández Sanchis D, Cuenca Zaldívar JN, Calvo S, Herrero P, Gómez Barrera M. Cost-effectiveness of upper extremity dry needling in the rehabilitation of patients with stroke. Acupunct Med. 2021;[Epub ahead of print]. 